\title{
A novel technique of measuring distance stereo- acuity using TNO chart and inverted telescope
}

\begin{abstract}
Purpose: To evaluate the feasibility of the TNO chart for the assessment of distance stereoacuity using an inverted telescope in normal subjects.
\end{abstract}

Methods: Stereoacuity of 30 emmetropic adult subjects was measured for near. The distance stereoacuity was measured using the TNO chart held at $40 \mathrm{~cm}$ and an inverted telescope (Galilean design, 26mm, 2X, Camma Inc. China) in front of the examinee's eyes.

Results: The near stereopsis ranged from 60" (seconds of arc) - 240" (Median 120"). Distance stereopsis ranged from 4.8" - 38.4". Distance stereoacuity could be measured in $14(47 \%)$ subjects.

Conclusion: It is possible to measure the distance with an inverted telescope and a TNO chart. Further improvisation is required to design an optimal telescope system with larger field, gradable zoom and measurable inter pupillary distance.

Keywords: stereoacuity, stereopsis, strabismus, telescope, TNO test, ocular alignment, intermittent exotropia, amblyopia, squint, stereo-test
Volume 7 Issue I - 2017

Mihir Kothari ${ }^{1,2,3}$

IJyotirmay Eye Clinic for Children and Adult Squint, Thane

${ }^{2}$ Bombay city eye institute and research center, India

${ }^{3}$ Mahatme eye hospital, India

Correspondence: Mihir Kothari, Jyotirmay Eye Clinic for Children and Adult Squint, Ocular Motility Lab and Pediatric Low Vision Center, 104, 105 Kalika Tower, Kolbad Road, Khopat, Thane West 40060I, Maharashtra, India, Tel +91222547808I, Emaildrmihirkothari@gmail.com

Received: May 16, 2017 | Published: May 25, 2017

\section{Introduction}

High grade stereopsis is the function of bifoveal fusion. Presence of fine stereopsis [stereoacuity $<40$ " (seconds of arc) on TNO test] essentially confirms an efficient and 'normal' binocular input. ${ }^{1}$ Measurements of Stereoacuity have been found useful in Monitoring the control of ocular alignment in intermittent strabismus viz. ${ }^{1,2}$ the intermittent exotropia, ${ }^{3-5}$ the intermittent esotropial and accommodative esotropia. ${ }^{6}$ Evaluating the functional outcome of strabismus surgery. ${ }^{7}$ Comparing the effects of early intervention in infantile strabismus syndromes viz. congenital esotropia. ${ }^{8}$ Screening of amblyogenic factors in children viz. anisometropia, significant ametropia and strabismus. ${ }^{9}$ In a strabismus clinic, measurement of distance stereopsis is particularly useful in the management of intermittent exotropia and esotropia of divergence paralysis type. This is mainly because reduction in the distance stereopsis is a sensitive sign of early deterioration of these strabismic disorders and generally makes a case for the surgical intervention. ${ }^{10}$ Various tests have been used for the measurement of stereoacuity for the near. ${ }^{11}$ Titmus/Wirt Fly test, Randot test and TNO test are among the more popular ones. For the distance stereopsis, the Mentor BVAT SG ${ }^{12}$ and Frisby Davis distance stereo test (FD2) ${ }^{13}$ are fairly accurate and versatile. However, the distance stereoacuity tests are not commonly available in the clinics and they are relatively expensive. In the present study, we have explored the option of using the TNO test that uses red-green random dot stereopairs (anaglyphics) with an inverted telescope to measure the global, crossed disparity induced, central stereopsis. To our knowledge this is the first paper in the literature to optically induce the distance using a telescope in an inverted fashion and combine its use with a near stereoacuity assessment chart for the measurement of distance stereoacuity.

\section{Subjects and methods}

This prospective cohort study was carried out in the Pediatric Ophthalmology and Strabismus department of a tertiary eye centre in the Western India. The emmetropic and orthophoric subjects in the age group of 20-35 years, visiting the Pediatric Ophthalmology or Strabismus clinic for their ward were included in the study. An optometry student performed a complete ophthalmic examination. Ocular motility evaluation was reconfirmed by a fellowship trained strabismologist. Once the subject was diagnosed as 'normal', an oral consent was obtained explaining the nature of the test and the need of the study. The eligible subjects were tested on the TNO test (TNO institute of Vision, Netherlands). Care was taken to hold the TNO chart at $40 \mathrm{~cm}$ (measured with a tailor's tape) in a brightly lit room by the same investigator in the same room conditions each time. The subjects were allowed enough time to recognize the 3 dimensional images in the test. Those who did not respond to the first three plates (implying the absence of gross stereopsis) were excluded from the study. The optometrist recorded the last correctly read plate by the subject.

At this stage a binocular telescope (Galilean design, 26mm, 2X, Camma Inc. China) was held in an inverted fashion in front of the red-green goggles by the subject. The inter pupillary distance (IPD) was adjusted by the subject, the test was run again and the response was recorded in the same fashion. Care was taken to maintain the test distance at $40 \mathrm{~cm}$ and the subjects were given enough time and explanations to complete the test. The study was approved by the institutional ethics committee.

\section{Results}

Of 30 subjects, $24(80 \%)$ were females. The age ranged from 21-35 years (mean 29, standard deviation 4.4). The near stereopsis ranged from 60"-240" (Median 120", 95\%CI 82"-117"). The distance stereopsis ranged from 4.8" ->38.4" (Median 38.4") in 14 (47\%) subjects. Sixteen (53\%) subjects reported inability to appreciate distance stereopsis.

\section{Discussion}

The ability in nearly half the subjects to appreciate distance stereopsis suggests the feasibility of using an appropriately designed 
inverted telescopic system for the assessment of distance stereopsis in combination with a near stereo chart. The TNO test is a popular test used to assess the near stereoacuity. In this test red-green anaglyphics are presented in a random dot stereopair. When viewed with the RedGreen filter (goggles) it induces the stereo effect that measures the global, central, crossed disparity threshold (central stereoacuity). Inversion of the chart (upside down) can test the stereoacuity for the uncrossed disparity. The advantages of TNO test over Titmus/Wirt fly test are two folds. 1) There are no monocular clues and 2) Red-Green colour optimizes the stereo contrast for the measurement (akin to the measurement of the Cone function with Photopic conditions- the optimization of Cone system).

By definition, stereoacuity is the smallest depth difference we can see, that is, a depth discrimination threshold. Thus stereoacuity can be thought of as the "resolving capacity" of stereopsis, much as visual acuity in the resolution limit of the spatial vision. In other words it is the measure of the horizontal binocular disparity that results from the images in each eye being formed on the closely spaced noncorresponding retinal points within Panum's fusional area. This disparity can be measured ${ }^{16}$ using the formula $n=2 a x / d 2$. Where $n$ is the angular stereo disparity in radians (to get the stereoacuity in arc seconds mulitply with 2,06,256), $2 \mathrm{a}$ is the inter pupillary distance (IPD), $\mathrm{d}$ is the fixation distance and $\mathrm{x}$ is depth interval. The fixation distance in the inverted telescope distance can be calculated using the formula $u=1 / v$ independently for each lens of the telescope (eye piece and the objective lens). The image of the first lens acting as the object for the second. Once the fixation distance is calculated, stereoacuity can be derived by measuring the IPD. The disparity value can also be calculated for any distance by multiplying the original value on TNO test chart by a factor $40 / \mathrm{d}$ where $\mathrm{d}$ is the viewing distance in $\mathrm{cm} .{ }^{17}$ The near stereoacuity measurements were similar to previously reported. Median distance stereo acuity in this study was 38.4" comparable to 29.6 " at 6 meters reported by Adams et al. ${ }^{17}$ and 37 " reported by Zaroff et al. ${ }^{18}$

However, Zanoni \& Rosenbaum ${ }^{19}$ using different distance stereo tests, have noted differences in the distance stereo-acuity values. Inability of 16 patients to appreciate the stereopsis necessitates the standardization of the telescopic system and control of accommodation and convergence during testing. The accommodation control would require adequate fogging in the telescopic system. While, the effect of convergence can be removed by presenting the stimulus for $100 \mathrm{~ms} .^{18}$ Presenting the stimulus for such a short time is certainly possible with the Frisby Davis charts and American Optical charts. While TNO test takes longer time for local stereopsis (Point to point matching of the corresponding random dots in the periphery) to occur and then global stereopsis (stereo averaging of the random points with the central disparity) to appear; hence patients should be allowed to take longer response time (at least $1000 \mathrm{~ms}$ per plate).

There are a few limitations of the TNO test. 1) It dissociates eyes, 2 ) it induces crowding phenomenon that reduces the visual acuity in the amblyopic eye more severely and thereby reducing the stereopsis, 3) TNO test induces colour rivalry between the two eyes and 4) the test produces chromatic aberration. These are also some of the reasons why the stereoacuity norms on TNO test are different (lower) than that with Titmus/Wirt Fly test. Similarly each of the distance Stereoacuity tests have their limitations and merits. We do believe that an inverted telescope system can be used with any other near stereoacuity chart. However, the test would need to be standardized, normative values need to be calculated and the repeatability needs to be assessed. So long as one is consistently using the same stereoacuity chart, the interpretation would remain the same.

The patients who did not appreciate stereoacuity using the inverted telescope in this study had excellent near stereoacuity. We believe the problem was their inability to adjust for the IPD and maintain the target within the visual filed. Patients with larger IPD $>68 \mathrm{~mm}$ had this problem. A wider field of telescope with adjustable IPD is likely to eliminate this problem. The effect of pictorial monocular clues was eliminated by the use of random dots and the use of telescopic system. However, the effect of convergence and accommodation though less likely to be clinically significant, may affect the measurements. In the present study we have essentially evaluated the central stereoacuity for the crossed disparity which is usually better than the uncrossed disparity induced stereoacuity. ${ }^{20}$ Nevertheless, the uncrossed disparity induced stereoacuity can be easily evaluated using the inverted telescope by inverting the TNO stereo chart.

\section{Summary}

Improvisation is required to fabricate a telescope with gradable zoom, adjustable IPD and aspheric lenses with larger field to be used with TNO chart in inverted fashion to measure distance stereo-acuity.

\section{Acknowledgements}

None.

\section{Conflicts of interest}

The authors declare there are no conflicts of interest.

\section{References}

1. Rutstein RP, Fuhr P, Schaafsma D. Distance stereopsis in orthophores, heterophores, and intermittent strabismics. Optom Vis Sci. 1994;71(7):415-421.

2. Read JC. Stereo vision and strabismus. Eye (Lond). 2015;29(2):214-224.

3. Kang KT, Lee SY. Relationship between control grade, stereoacuity and surgical success in basic intermittent exotropia. Korean J Ophthalmol. $2015 ; 29(3): 173-177$

4. Singh A, Sharma P, Singh D, et al. Evaluation of FD2 (Frisby Davis distance) stereotest in surgical management of intermittent exotropia. $\mathrm{Br}$ J Ophthalmol. 2013;97(10):318-1321.

5. Feng X, Zhang X, Jia Y. Improvement in fusion and stereopsis following surgery for intermittent exotropia. J Pediatr Ophthalmol Strabismus. 2015;52(1):52-57.

6. Castro-Vite OI, Vargas-Ortega AJ, Aguilar-Ruiz A, et al. Sensorial status in patients with pure accomodative esotropia. Arch Soc Esp Oftalmol. 2016;91(12):573-576.

7. Goseki T, Ishikawa $H$. The prevalence and types of strabismus, and average of stereopsis in Japanese adults. Jpn J Ophthalmol. 2017;61(3):280-285

8. Ing MR, Okino LM. Outcome study of stereopsis in relation to duration of misalignment in congenital esotropia. J AAPOS. 2002;6(1):3-8.

9. Farvardin M, Afarid M. Evaluation of stereo tests for screening of amblyopia. Iranian Red Crescent Medical Journal. 2007;2007:5-10.

10. Hatt SR, Gnanaraj L. Interventions for intermittent exotropia. Cochrane Database Syst Rev. 2013;31(5):CD003737.

11. Marsh WR, Rawlings SC, Mumma JV. Evaluation of clinical stereoacuity tests. Ophthalmology. 1980;87(12):1265-1272. 
12. Yildirim C, Altinsoy HI, Yakut E. Distance stereoacuity norms for the mentor B-VAT II-SG video acuity tester in young children and young adults. J AAPOS. 1988;2(1):26-32.

13. Holmes JM, Birch EE, Leske DA, et al. New tests of distance stereoacuity and their role in evaluating intermittent exotropia. Ophthalmology. 2007;114(6):1215-1220

14. Singman EL, Matta NS, Silbert DI, et al. Comparison of the INNOVA Visual Acuity System Stereotest with the Frisby-Davis 2 Stereotest for the Evaluation of Distance Stereoacuity. Binocul Vis Strabolog Q Simms Romano. 2013;28(2):78-83.

15. Steinman SB. Stereopsis. Foundations of Binocular Vision: Clinical Perspectives. In: Steinman SB, Steinman BA, editors. 1st edn. New Jersey, USA. McGrawhill Company; 2000:173-234.
16. Okuda FC, Apt L, Wanters BS. Evaluation of the TNO random-dot stereogram. Am Orthopt J. 1977;27:124-130.

17. Adams WE, Hrisos S, Richardson S, et al. Frisby Davis distance stereoacuity values in visually normal children. $\mathrm{Br} J$ Ophthalmol. 2005;89(11):1438-1441.

18. Zaroff CM, Knutelska M, Frumkes TE. Variation in stereoacuity: normative description, fixation disparity, and the roles of aging and gender. Invest Ophthalmol Vis Sci. 2003;44(2):891-900.

19. Zanoni D, Rosenbaum AL. A new method for evaluating distance stereo acuity. J Pediatr Ophthalmol Strabismu. 1991;28(5):255-260.

20. Larson WL. An investigation of the difference in stereoacuity between crossed and uncrossed disparities using Frisby and TNO tests. Optom Vis Sci. 1990;67(3):157-161. 